\title{
Isolated delusion: an uncommon feature in idiopathic Parkinson's disease
}

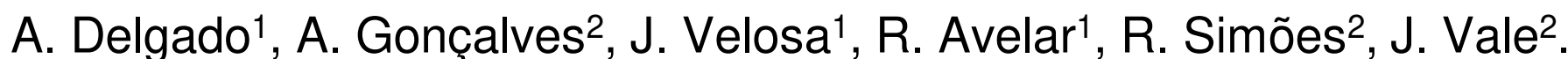
ÂNGELO

${ }^{1}$ Hospital Beatriz Ângelo, Psychiatry, Loures, Portugal.

${ }^{2}$ Hospital Beatriz Ângelo, Neurology, Loures, Portugal.

\section{Objectives:}

Characterize a subgroup of Parkinson's Disease (PD) patients with isolated delusions (ID).

\section{Background:}

PD patients are particularly prone to develop psychotic symptoms due to the disease itself and to dopaminergic treatments. Visual hallucinations, with or without delusions, are the classic manifestation, mainly associated with disease progression and cognitive deterioration. However, a distinct subset may develop ID.

\section{Materials and Methods:}

PD patients followed by the authors between 01.01.2014 and 31.01.2018 at a movement disorders clinic were identified using the ICD9 code 3320. Charts were retrospectively reviewed to identify patients with ID. Neurological and Psychiatric interviews were performed, including MDS-UPDR, MoCA, NPI, SCOPA-PC, HAM-D.

\begin{tabular}{|c|c|c|c|c|c|c|c|c|}
\hline & $\mathrm{N1}$ & N2 & N3 & $\mathrm{N} 4$ & N5 & N6 & N7 & N8 \\
\hline Sex & $F$ & $F$ & $F$ & $\mathrm{~F}$ & $\mathrm{~F}$ & $\mathrm{~F}$ & $\bar{M}$ & $M$ \\
\hline $\begin{array}{l}\text { Age of DP } \\
\text { diagnosis }\end{array}$ & 59 & 59 & 61 & 61 & 64 & 33 & 50 & 61 \\
\hline $\begin{array}{l}\text { DP duration } \\
\text { (years) }\end{array}$ & 7 & 8 & 21 & 5 & 5 & 5 & 14 & 4 \\
\hline $\begin{array}{c}\text { Psychiatric } \\
\text { history }\end{array}$ & No & No & No & No & No & No & $\begin{array}{l}\text { Depression } \\
\text { with } \\
\text { psychotic } \\
\text { symptoms }\end{array}$ & No \\
\hline Delusion & Paranoid & Paranoid & Jealousy & Paranoid & Jealousy & Jealousy & Paranoid & Paranoid \\
\hline $\mathrm{H} \& \mathrm{Y}$ & 2 & 2 & 3 & 2,5 & 2,5 & 1,5 & 2 & 2 \\
\hline MoCA & 23 & 21 & 25 & 14 & 23 & 29 & 24 & 14 \\
\hline NPI & $\begin{array}{c}\text { Delusion } \\
9 / 15\end{array}$ & 0 & 0 & $\begin{array}{c}\text { Delusion } \\
15 / 15\end{array}$ & $\begin{array}{c}\text { Delusion } \\
4 / 15\end{array}$ & $\begin{array}{c}\text { Delusion } \\
8 / 15\end{array}$ & $\begin{array}{c}\text { Delusion } \\
9 / 15\end{array}$ & 0 \\
\hline $\begin{array}{l}\text { Last change } \\
\text { before } \\
\text { delusion }\end{array}$ & $\uparrow$ Ropinirole & $\begin{array}{c}\uparrow \\
\text { Ropinirole }\end{array}$ & $\begin{array}{c}\text { Started } \\
\text { Ropinirole }\end{array}$ & $\begin{array}{l}\uparrow \text { Levodopa } \\
\text { (LD) }\end{array}$ & $\begin{array}{c}\uparrow \\
\text { Ropinirole }\end{array}$ & $\begin{array}{l}\text { Started } \\
\text { Ropinirole }\end{array}$ & $\uparrow$ Levodopa & $\begin{array}{c}= \\
\text { Ropinirole }\end{array}$ \\
\hline $\begin{array}{l}\text { Course of } \\
\text { action }\end{array}$ & $\begin{array}{l}\text { Stop } \\
\text { ropinirole, } \\
\text { started } \\
\text { quetiapine }\end{array}$ & $\begin{array}{l}\text { Stop } \\
\text { ropinirole, } \\
\text { started } \\
\text { quetiapine }\end{array}$ & $\begin{array}{l}\text { Stop } \\
\text { ropinirole, } \\
\text { started } \\
\text { quetiapine }\end{array}$ & $\begin{array}{c}\downarrow L D, \\
\text { started } \\
\text { quetiapine } \\
\text { then } \\
\text { clozapine }\end{array}$ & $\begin{array}{l}\text { Stop } \\
\text { ropinirole, } \\
\text { started } \\
\text { quetiapine }\end{array}$ & $\begin{array}{l}\text { Stop } \\
\text { ropinirole, } \\
\text { started } \\
\text { quetiapine }\end{array}$ & $\begin{array}{c}\text { Stop } \\
\text { ropinirole, } \\
\downarrow \text { LD, } \\
\text { started } \\
\text { aripiprazole }\end{array}$ & $\begin{array}{c}\text { Stop } \\
\text { ropinirole }\end{array}$ \\
\hline Outcome & $=$ & $\begin{array}{c}\text { Total } \\
\text { remission }\end{array}$ & $\begin{array}{c}\text { Total } \\
\text { remission }\end{array}$ & $=$ & $=$ & Improved & Improved & $\begin{array}{c}\text { Total } \\
\text { remission }\end{array}$ \\
\hline
\end{tabular}

\section{Results and Conclusions:}

Of 127 patients with PD, 10 had ID and 8 were interviewed: 2M:6F, aged 66,1 \pm 11 , age at onset of PD 58 years, mean duration of disease of 8 years, Hoehn \& Yahr 2. Levodopa (LD) equivalent dose was 530mg and all except one were on dopamine agonists (DA) when delusions started. Mean MoCA score was 23. Delusions were paranoid (5) or jealousy (3) in nature. With reduction/interruption of DA alone or with institution of antipsychotics, 5 improved or resolved. As described in the literature, these PD patients who developed ID were younger, in an early stage of disease progression, non demented, and there was a clear relation to DA, with remission only in a small sample. Facing a PD patient with ID should lead to a careful therapeutic review as reduction/withdrawal of the DA may be enough to improve symptoms. Awareness, active screening and multidisciplinarity are key to early recognition and treatment. 